\title{
Da Brindisi a Lampedusa: le migrazioni via mare nella cassa di risonanza della popular music italiana
}

\author{
Nora MOLL (Roma)
}

\section{Summary}

From the landing of thousands of Albanese people on the Apulian coast in 1991 to the daily arrival of refugees on the Calabrian and Sicilian coasts since 2000, immigration by sea and the lifestories of the countless victims of this situation have become part of the 'public awareness' in Italy. To narrate the tragedies we are witnessing day by day, different communicational strategies have been adopted, depending on the respective media and on the political objectives pursued by individual 'speakers'. The attention writers, film directors, and artists have payed to the recent migration movements towards Italy has gradually given a more profoundly human dimension to the phenomenon and has brought the public closer to the individual tragedy. Italian popular music in particular has actively contributed to establishing an artistic counter-discourse, which will be discussed in the present article. Examples of different musical genres will be analysed, covering the time frame in question, whereas the methodology pursued will be that of Imagology and that of Cultural Studies.

Ecco la nostra vita impastata senza lievito, pane mandato sopra i volti delle acque.

(Erri De Luca)

In fondo al mare, in fondo al mar profondo, ci lascio il canto mio, che non consola, per chi è partito e si è perduto al mondo.

(Gianmaria Testa)

Da quando, l'8 marzo 1991, circa ventimila migranti albanesi raggiunsero il porto di Brindisi, stipati fino all'inverosimile sulla nave mercantile Vlora, l'immaginario collettivo italiano è stato fortemente segnato dall'arrivo via mare di masse di migranti. Si tratta di masse percepite, in un primo momento, come uniformi, anonime, difficilmente ricollocabili e 
minacciose, protagoniste di eventi che solo in tempi più recenti sono stati narrati in maniera più differenziata, e a partire dal punto di vista di chi in quei mesi fuggiva dall'Albania. ${ }^{1}$ Dieci anni dopo, invece, si intensificarono gli sbarchi sulle coste calabresi e siciliane, iniziati già alla fine degli anni ' $80 .{ }^{2}$ Questa volta si tratta di persone provenienti dall'Africa, dal Maghreb, dall'Africa subsahariana, dal Corno d'Africa e quindi dalle ex-colonie italiane Eritrea e Somalia. In seguito, dopo l'inizio nel 2011 delle rivolte nei paesi arabi e con il loro trasformarsi in guerre civili nonché internazionali, si sarebbe intensificato l'arrivo, sulla tratta mediterranea, di maghrebini e, successivamente, di profughi siriani. Ancora una volta parliamo di masse per lo più senza un preciso volto, di migranti e profughi che raggiungono cifre altissime e di difficile registrazione, e la cui condizione drammatica scuoterà la coscienza collettiva in alcune date poi divenute simboliche: il 28 marzo 1997, quando la motovedetta albanese Katër I Radës, con più di cento persone a bordo, affondò a seguito dello speronamento da parte di una nave della Marina Militare Italiana; il funerale di Stato di tredici naufraghi somali nel Campidoglio romano, il 24 ottobre $2003,{ }^{3}$ e il naufragio del 3 ottobre 2013 davanti alle coste dell'isola di Lampedusa, in cui persero la vita 388 persone, mentre i superstiti furono 155 . Tuttavia, si tratta di tragedie che si ripeteranno fino ad assuefare il pubblico dei mass media a resoconti di naufragi con decine e centinaia di morti in mare al giorno, e che nel discorso giornalistico e politico italiano sono spesso associate a parole-fantasma ${ }^{4}$ quali 'emergenza immigrazione', 'invasione' da parte di 'clandestini', e a slogan di stampo sensazionalistico e/o xenofobo. ${ }^{5}$

Difatti, a partire dal 2011 l'isola di Lampedusa, porto d'approdo principale di imbarcazioni di fortuna, è diventata uno dei massimi simboli europei dell'arrivo di migranti e profughi maggiormente colpiti dalla fame e dalla guerra, sfruttati dai trafficanti di uomini ma anche poco protetti dalla legislazione internazionale. Non solo: l'approdo via mare, nel corso degli anni si è trasformato in Italia in una sorta di 'icona mediatica' dal forte impatto visivo ed emotivo, che ha sovrastato l'intero discorso circa il fenomeno migratorio. ${ }^{6} \mathrm{La}$ costruzione sociale della realtà e dell'immaginario, rispetto a tale fenomeno ormai decennale, è stata quindi significativamente improntata sul detto e sul dicibile mediatico, laddove i mass media italiani si configurano come una sorta di 'arena' il cui agenda setting risponde volta per volta a delle precise esigenze politiche (Bruno 2014, 62-67). ${ }^{7}$ In questo contesto, la stigmatizzazione di migranti e profughi come 'clandestini' e la criminalizzazione degli stessi ha raggiunto dei picchi, ben tracciabili, ${ }^{8}$ nel 2008 , anno al quale risalgono gli accordi bilaterali tra l'allora presidente del consiglio Silvio Berlusconi e l'allora presidente libico Mu'ammar Gheddafi. La conseguente politica di respingimenti, dichiarati solo nel 23 febbraio 2012 come illegali dalla Corte Europea dei Diritti Umani, rappresenta difatti uno dei capitoli più bui dell'intera storia dell'immigrazione verso l'Italia, capitolo sul quale è stato fatto luce in anni più recenti in una serie di ricerche e docufilm. ${ }^{9}$

Rivolgendoci a questo punto e dopo le dovute premesse al nostro oggetto d'analisi, ossia ad alcuni brani di musica italiana di vario genere, che tematizzano tali viaggi della speranza di migranti attraverso le rotte del Mediterraneo, sarà utile mettere in luce fin da subito quali siano le domande poste a questo nostro piccolo campione musicale. In un primo luogo, 
infatti, ci si è chiesti in che maniera la popular music italiana partecipasse lungo l'arco di tempo preso in considerazione, e insieme ad altre forme di rappresentazione artistica, alla costruzione di un immaginario che vuole prendere le distanze dalla retorica politica e dal linguaggio dei mass media. Allo stesso tempo, era importante riflettere sul rapporto tra le modalità linguistiche e retoriche con cui questa difficile tematica viene affrontata, e il genere musicale nel quale la singola canzone va inquadrata: nel nostro caso il genere pop, il brano di un cantastorie, il folklore del sud Italia e l'hip hop. Infine, è parso significativo verificare se la singola canzone, o il singolo brano, fosse frutto di un'iniziativa isolata oppure se essa potesse essere inquadrata in un progetto più generale, all'insegna dell'engagement per la causa e i diritti dei migranti e/o profughi, e se, infine, facesse leva sulla collaborazione con altri artisti o intellettuali, di origine non solo italiana, nello spirito della creazione di una comunità artistica e narrativa ${ }^{10}$ che offrisse degli stimoli ulteriori ad una visione più ricca $\mathrm{e}$ differenziata del fenomeno in questione.

All'interno di tale percorso critico-analitico, sono stati utilizzati gli strumenti metodologici di varie discipline, e in particolare quegli elaborati dall'imagologia letteraria, dagli studi culturali e dalla narratologia, ancora prima che dagli studi testuali sulla canzone. Senza voler ambire, in questa sede, alla discussione delle specifiche posizioni teoriche riguardanti queste stesse discipline, si è così cercato di collocare il fenomeno musicale nel suo contesto culturale e socio-politico, trattandolo quindi come parte fondamentale di un più generale discours culturel: come sua componente significativa oppure, in altre parole, sua 'cassa di risonanza'.

\section{Come diventare un turista: Samuele Bersani e l'immagine dell'immigrato albanese}

Nel nostro primo esempio, una canzone pop intitolata "Barcarola albanese"11 di Samuele Bersani, difatti si tratta di un brano che segue di pochi anni il primo approdo di migranti albanesi, e di un'escursione unica nell'ambito tematico della migrazione, da parte del suo autore. In netto contrasto con le immagini comunemente associate agli sbarchi di persone provenienti dalla sponda orientale del mar Mediterraneo, di forte impatto perché legate all'idea di masse ingestibili e anonime, il cantautore originario di Rimini vi sceglie di affidare la responsabilità del racconto ad un 'io' narrante (o per meglio dire un 'io' cantante), che, nel songworld ${ }^{12}$, è giustappunto arrivato dall'Albania nella sua barchetta a remi. La prima strofa della canzone bipartita, infatti, inizia (dopo la intro di piano e archi) con limpide sequenze in Fa maggiore, e con facile rima interna nel primo e nel quinto verso, associata alle rime assonanti dei versi pari:

Vado veloce sopra questa noce

fuori pericolo,

le onde sono dei vetri, alte dei metri 
però le supero.

Il sole si sposta e già si vede la costa

qui dal binocolo.

Puntare dritto in avanti con due stuzzicadenti

Remare al massimo!

Queste prime battute narrano quindi le sensazioni provate dall'io' cantante durante una traversata, abbozzano con brevi pennellate i suoi pericoli ("le onde sono dei vetri, alte dei metri”) e la speranza di giungere a riva in salvezza. Speranza che si avvera, sul piano diegetico del song, nel successivo refrain, le cui parole disegnano altre e diverse speranze del migrante, appena giunto in Italia: quella di essere non solo salvo, ma anche libero (in una dimensione al plurale che emerge solo in questo momento e che include un interlocutore non meglio specificato), e di poter circolare come un turista qualsiasi. Una speranza, questa, che l'ultima battuta del refrain fa svanire repentinamente: il migrante in questione è nell' illegalità, e teme quindi le forze dell'ordine:

Per arrivare fino a Brindisi

pagherò,

saremo liberi per sempre,

potremo visitare Rimini.

Vieni via! Ci sono i vigili...

Ora, l'inserimento della nota stonante dell'arrivo dei vigili nell'idillio dello sbarco - nota tematica che viene rafforzata da repentine scalate espressivo-vocali - $\mathrm{fa}$ da segnale per un'altra stonatura sul piano del discorso, del resto ben enunciata già dal titolo della canzone di Bersani. In quest'ultimo, il termine 'barcarola' rimanda (con evidente ironia svelata sin da subito dall'accostamento con l'aggettivo 'albanese') ad un genere vocale tradizionale, caratterizzato dal canto dai ritmi moderati dei gondolieri veneziani, e confluito a varie riprese anche nella musica classica (da Chopin ad Offenbach); un genere che, in sostanza, viene declinato solitamente nei modi di un idillio romantico. Già dopo le prime battute del testo, e sullo sfondo di un immaginario condiviso con l'ascoltatore del brano, è però ben chiaro che qui non si tratti di un idillio, e che non siamo di fronte a traversate romantiche, bensì a viaggi della speranza finiti in alcuni casi in maniera letale (difatti, e forse per coincidenza, la canzone fu riproposta nel successivo album Samuele Bersani, uscito nel 1997 ossia nello stesso anno dell'affondamento della Katër I Radës). Non solo: al di là dell'uso improprio e ridicolizzante del termine 'vigili' nel refrain (al posto di un più appropriato e realistico 'poliziotti' o 'carabinieri' e dettato con tutta probabilità da esigenze di rima), nella seconda strofa di "Barcarola albanese" si chiarisce ulteriormente che la narrazione autodiegetica qui usata, per sua natura più empatica del relato in terza persona, non implichi necessariamente e tout court un cambiamento del punto di vista sulla questione migratoria: 


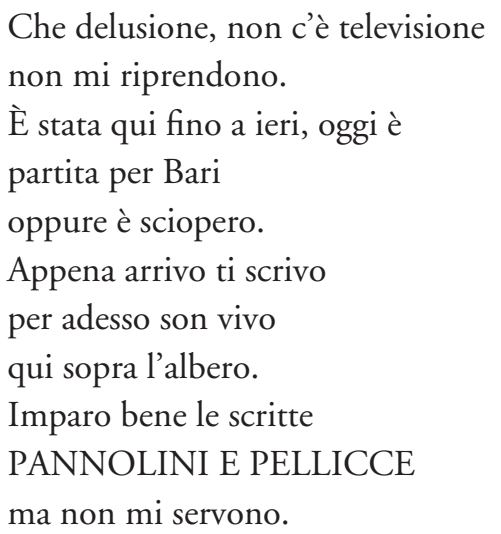

Difatti, come si può notare, la dramatis persona della breve fabula è un personaggio ingenuo, che crede di poter arrivare in Italia da turista, che vuole essere ripreso dalla $\mathrm{tv}^{13} \mathrm{e}$ che per di più si è rifugiato su un albero, imparando a memoria le scritte dei negozi e sognando di essere libero. Quel che ne risulta è una caricatura del migrante albanese 'tipo', costretto in una posa e in un pensiero eccessivamente ingenui e finanche ridicoli. Possiamo quindi parlare di una presa di distanza dell'autore implicito del testo-in-musica rispetto allo stesso personaggio al quale il cantautore presta la sua voce. Attraverso tale breve ed ironico schizzo di una interiorità immaginata, la canzone di Bersani, più che avvicinare realmente il suo pubblico alle problematiche legate al contesto storico-sociale in questione, si inserisce quindi sul piano semantico nell'ambito di una definizione dell'Altro già pre-vista dall'immaginario collettivo configurato dai mass media. In altre parole, nonostante il cambio di prospettiva, il testo di Bersani non sfugge alla polarizzazione tra il noi (civile, democratico, superiore) e il loro (inferiore perché ingenuo, comunista, ignorante), che è solo l'altra faccia della medaglia di atteggiamenti più esplicitamente fobici, espressi in quegli anni dalla cultura d'arrivo.

\section{L'arrivo degli “innumerevoli”: il Canzoniere Grecanico Salentino e i suoi riferimenti letterari}

Molto diverso, sul piano dello stile musicale nonché su quello testuale, il brano "Solo andata”, del Canzoniere Grecanico Salentino (2014), composto da Daniele e Mauro Durante su parole di Erri De Luca (2005). Appare intanto singolare la scelta da parte del gruppo di musica tradizionale salentina, attivo dal 1975 e i cui sette membri sono protagonisti dell'ormai quasi ventennale manifestazione di cultura popolare La notte della Taranta ${ }^{14}$, di utilizzare un testo letterario. In questo caso, la scelta cade su un testo dal tema migratorio, di forte impatto e di chiaro engagement. Tale scelta può essere quindi già ad un primo sguardo interpretata come il desiderio, da parte del gruppo, di prendere apertamente posizione, associandosi all'engagement di Erri De Luca e collaborando con lui in diversi spettacoli e manifestazioni musicali. ${ }^{15}$ 
Nello specifico, Durante padre e figlio mettono in musica le ultime due strofe di un poe$\mathrm{ma}$ in cui lo scrittore cerca di conferire una voce alle masse di migranti giunti da oltremare, di attribuire sul piano poetico e fittizio agli "innumerevoli" che "aumentano se contati" dei desideri, una memoria, una visione riguardo quel 'voi' rappresentato dalla cultura d'arrivo (e che in realtà si identifica sul piano della ricezione con il 'noi' che leggiamo e ascoltiamo). Anche qui, come nel testo della canzone di Bersani, siamo quindi di fronte all' uso della prima persona, ma questa volta la scelta cade sin dalle prime battute sul plurale collettivo di un coro immaginario, ben tradotto nell'arrangiamento di voci soliste alternate con sequenze a cappella, a cinque voci: ${ }^{16}$

Siamo gli innumerevoli, raddoppia ogni casella di scacchiera

lastrichiamo di corpi il vostro mare per camminarci sopra.

Non potete contarci, se contati aumentiamo

figli dell'orizzonte che ci rovescia a sacco.

Nessuna polizia può farci prepotenza

più di quanto già siamo stati offesi.

Faremo i servi, i figli che non fate,

le nostre vite saranno i vostri libri di avventura.

Portiamo Omero e Dante, il cieco e il pellegrino,

l'odore che perdeste, l'uguaglianza che avete sottomesso.

L'estratto qui riportato, così come tutto il componimento poetico di De Luca, è disseminato di metafore (ad esempio "figli dell'orizzonte", "l'odore che perdeste" nella prima, e "noi siamo il rosso e il nero della terra", "un oltremare di sandali sfondati" nella seconda strofa) che conferiscono ad esso delle note arcaizzanti. Affianco a tali valenze figurative delle scelte lessicali, che trovano il loro correlativo oggettivo nelle scelte musicali (l'uso di strumenti quali il flauto dolce, il tamburello e la tammorra, ad esempio), sono da notare i toni profetici di questi versi, i quali creano una rete di rimandi ad altri testi apertamente impegnati nella denuncia delle diseguaglianze sociali. Così, l'aggettivo "offesi" fa da eco al vittoriniano "genere umano offeso"17, ma ricorda anche tutta una letteratura che va da Fanon a Sartre e a Gramsci, fino a Pier Paolo Pasolini e in particolare alla sua famosa poesia "Profezia" (del 1962), ${ }^{18}$ ossia autori che hanno descritto ed analizzato in termini lucidi e allo stesso tempo suggestivi la grande spaccatura che divide il mondo sviluppato e 'civile' e quello dei "dannati dalla terra" (Fanon 2007), del Meridione d'Europa e del mondo Sud. Nella strofa seguente, poi, $\mathrm{i}$ toni profetici si addenseranno in un monito crescente e finanche minaccioso: intanto, si riprende sul piano semantico la dimensione epica dell'iniziale "siamo gli innumerevoli" ("Da qualunque distanza arriveremo a milioni di passi / noi siamo i piedi e vi reggiamo il peso", recitano i primi due versi della seconda strofa), e a seguire incalzano i riferimenti al ruolo di 'subalterni' (per usare il ben noto concetto gramsciano) al quale i migranti del mondo Sud sono costretti ("Spaliamo neve, pettiniamo prati, / battiamo tappeti, raccogliamo il pomodoro e l'insulto"). Si tratta di un crescendo, sul piano semantico-testuale ma anche 
musicale, nel quale emerge con chiarezza l'intenzione di Erri De Luca, e insieme a lui del gruppo salentino, di fare leva sul concetto di dignità originaria, arcaica, di cui sono portatori i migranti, una dignità che la cultura d'arrivo avrebbe perduta, dimenticando anche la comune radice mediterranea (che nella 'profezia' di Pasolini, scritta negli anni sessanta sullo sfondo della guerra di liberazione coloniale algerina e della posizione sartriana in merito, porterebbe ancora al reciproco riconoscimento, tra i 'dannati della terra' di entrambe le sponde).

Il componimento culmina infine nel riferimento, ben esplicito e carico anch'esso di assonanze pasoliniane, alla figura di Cristo, attraverso una citazione libera che lascia la voce direttamente al Salvatore ("Uno di noi, a nome di tutti, ha detto: 'Non vi sbarazzerete di me. / Va bene, muoio, / ma in tre giorni resuscito e ritorno"”): una scelta questa che è ben in linea con la poetica del più maturo De Luca, che per anni è stato anche traduttore dall'ebraico di capitoli dell'Antico Testamento e della Torah, nonché autore di diversi libri che vi si ispirano. Si tratta quindi di una chiusura testuale solenne, seguita da un'emozionante accelerazione ritmica e di intensità sonora più tipiche della 'pizzica tarantata', che vuole senz'altro rafforzare ulteriormente il monito rivolto ad un'Italia e un'Europa che non accoglie, ma che respinge i figli - più dignitosi proprio perché più poveri e più semplici - di queste terre lontane: una chiara (e per alcuni scomoda) presa di posizione che le parole finali recitate dallo stesso Erri De Luca nel videoclip ufficiale (firmato Alessandro Gassman) non fanno che parafrasare ulteriormente. ${ }^{19}$

\section{Dentro la barca: il cantastorie Mauro Geraci}

Anche nel componimento seguente, la presa di posizione da parte dei boat people in viaggio verso l'Europa, si unisce a delle scelte musicali e testuali che richiamano le tradizioni italiane regionali. In questo caso si tratta della canzone "Lampedusa" (del 2014) di Mauro Geraci, un antropologo messinese che da anni pratica l'antico mestiere popolare dei cantastorie. Si tratta di una forma musicale-testuale tradizionalmente legata ai fatti di cronaca, ai temi passionali dalle forti tinte, ma anche, specie nel caso di autori come Franco Trincale (al quale Geraci apertamente si ispira ${ }^{20}$ ), alla denuncia politica e sociale. In un primo momento legato all'estemporaneità e alle manifestazioni popolari, dagli anni '60 in poi sono stati incisi dei dischi con questo genere di canzoni, da artisti quali Paolo Garofalo, Vito Santangelo, lo stesso Trincale e altri. In tempi più recenti, invece, i cantastorie come lo stesso Geraci hanno usato anche lo strumento del web per raggiungere un pubblico più ampio, creando dei blog e pubblicando le tracce audiovisive delle loro canzoni su grandi piattaforme internet. ${ }^{21}$

Più degli altri cantanti e cantautori da me esaminati, Geraci in questo suo componimento in lingua siciliana riesce ad esprimere l'orrore della traversata sul mar Mediterraneo, e, con più intensità degli altri, a far immedesimare l'ascoltatore nei sogni ad occhi aperti, nelle paure e nelle speranze dei migranti. Sin dalle prime battute della canzone (strutturata in tre blocchi da tre quartine endecasillabe a rime alterne, più refrain da otto versi quinari, l'uno) 
il tono scelto dall'antropologo messinese è privo di pathos soggettivo; egli mantiene invece una distanza narrativa la cui solennità viene accentuata dalla scelta del ritmo in quattro quarti e da una tonalità dal timbro sostenuto, in Mi minore (alla quale subentra il Re maggiore dei ritornelli in tre quarti, il cui andamento più sollevato è in realtà in contrasto con il contenuto semantico dei rispettivi testi):

Lu cielu supra a navi si fa strittu guardatu di mill'occhi a la diriva, l'avutri milli non hannu dirittu, pi d'iddi l'universu è nta la stiva.

Tripoli è ormai luntana e non si vidi, non ci su' stiddi e non c'è cchiù la luna ma cu sta sutta veru non ci cridi, pensa passatu 'u mari a la furtuna.

Lu ventu è friddu e ognunu si li strinci li vrazza nta li spaddi pi cuperta, la fami ferma, 'a siti e comu sfinci affrunta ora l'urtima trasferta.

...mari e disertu

lu stissu sunnu, siccanu a vucca, la peddi e l'ossa, siccanu 'a storia, puru li nomi chiddi ca 'u passanu non hannu cchiù.

Attraverso delle scelte lessicali e figurative molto efficaci, e sullo sfondo di un pacato accompagnamento chitarristico, Geraci dipinge quindi lo scenario di una traversata notturna, in cui il cielo appare "stretto" e le sponde libiche lontane; le stelle ("stiddi") e la luna sovrastano il mare aperto, il "vento freddo" tormenta i profughi stipati nella nave, che si stringono le spalle per scaldarsi ("li vrazza nta li spaddi pi cuperta"), e che "come sfingi" affrontano "l'ultima trasferta". Nonostante la scelta della terza persona (alla quale solo nel secondo refrain subentrerà un generico tu), la focalizzazione della narrazione è a tratti interna (il cielo "si fa stretto" proprio perché visto a partire dalla nave), in modo tale da sollecitare l'immedesimazione da parte dell'ascoltatore, e a tratti assente, al fine di poter pronunciare dei commenti di tipo morale. Difatti, sin dalle prime battute della canzone, i migranti via mare sono definiti come dei 'senza diritti' ("l'avutri milli non hannu dirittu"), mentre nell'ultima sezione 
tristrofica la denuncia di stampo politico si fa più esplicita. Si tratta di una denuncia rivolta prima di tutto ai grandi d'Europa che si riuniscono a Bruxelles, oltre che al governo italiano, responsabile delle condizioni spesso disumane in cui i profughi versano nei Centri di Permanenza Temporanea (termine che qui spicca nella sua rigidità fonetica anche per l'uso isolato dell'italiano):

E Lampedusa pari fussi Europa... carzaru duru è pi st' innuccenti, dd'occhi sgranati tisi all'orizzonti ora su' chiusi a chiavi nta li centri.

Centri di permanenza temporanea unni li besti ammassanu a lu stazzu, lu suli a picu li testi trapana, lu granni cu lu nicu nesci pazzu.

Mentri a Bruxelles lu Miditirraneu na cartulina pari, na bannera, pittattu azzurru è ogni lituraneu e luciunu li stiddi a ogni riviera.

...pari l'Europa

all'orizzonti

'nveci è un canali

senza Caronti.

A Lampedusa

trova cu sbarca

sulu na madri

chi porta l'acqua.

Lampedusa, come porta d'ingresso di un'Europa raggiunta attraverso gli stenti descritti più in dettaglio nel corso della seconda sezione della canzone, ${ }^{22}$ è quindi solo un inganno, e il canale di Sicilia l'ingresso per un nuovo inferno, nel quale solo la pietà e l'iniziativa del singolo ("na madri / chi porta l'acqua"), e non dei governi, può portare sollievo. Una conclusione, questa, di certo molto pessimistica, alla quale Geraci giunge mettendo in gioco un linguaggio non solo realistico-descrittivo, bensì anche simbolico: e a questo proposito si noti soprattutto l'uso dell'immagine delle stelle, che da simbolo (ben presto oscurato) di speranza per un futuro migliore passa ad assumere il suo valore di icona dell'Unione Europea, oscurata e inaccessibile anch'essa, per chi arriva dall'altra parte del mare. Anche lo stesso mare, nella prospettiva narrativa oscillante interna-esterna del testo di Geraci, percorre una serie di valenze, che contribuiscono a mettere in luce i paradossi della condizione degli umani nel 
nostro oggi: da mare che, insieme al deserto, "sicca[nu] a vucca” (nella prima parte), al "mare 'e muri" (della seconda), fino a giungere al contrasto stridente con il mar Mediterraneo voluto dall'Europa più ricca come "cartolina". Una cartolina, sembra voler concludere Geraci, sulla quale la morte non deve essere rappresentata, per non turbare la coscienza di chi gode della 'tavola apparecchiata' (“cunzata”).

\title{
Dalla parte delle madri: Fiorella Mannoia con “In viaggio"
}

Passando da questo esponente di una forma musicale dal pubblico molto limitato (ma potenzialmente più ampio, grazie all'uso dei mezzi di diffusione digitali), ad un esempio di musica pop, ossia alla canzone "In viaggio" di Fiorella Mannoia (2012), cambiano difatti, e radicalmente, non solo le sonorità, bensì anche il linguaggio, i motivi e il grado di esplicitezza con cui viene affrontato il tema migratorio. La canzone è a firma di una delle grandi signore della popular music italiana, che ha al suo attivo ben 39 album, diversi dei quali dal vivo. Mannoia è sempre stata interprete, non autrice: va infatti notato che solo con l'album Sud, la cantante romana appaia per la prima volta come autrice, in particolare, per l'appunto, del testo di "In viaggio" (oltre che di "Se solo mi guardassi"). Da anni impegnata in cause civili e sostenitrice della ONG Emergency, nel tempo le sue canzoni hanno assunto in misura crescente toni filantropici, facendo appello al sentire umano e alla partecipazione emotiva nei confronti di realtà e storie non solo della Gente comune (come si intitola un suo album del 1994), bensì dell'incontro tra questa stessa 'gente', di noi tutti, con le culture extraeuropee (tema presente in particolare in Se solo mi guardassi). Difatti, da diverso tempo la cantante è in dialogo con artisti e scrittori migranti, una circostanza che le ha conferito un ruolo speciale in numerose iniziative di associazioni e di ONG attive nella salvaguardia dei diritti di migranti e profughi. ${ }^{23}$ Sul piano artistico-musicale, tali collaborazioni sono peraltro palpabili nella partecipazione, nello specifico al suo album del 2012, di cantanti e musicisti provenienti dal mondo Sud, tra cui Badara Seck e Kaw Dialy Maly Sissoko, entrambi griot e suonatori di cora senegalesi.

A discapito del suo titolo, la canzone di Mannoia, in questo contesto di canzoni sul viaggio migratorio via mare, sembra in realtà fuori tema, perché difatti nelle sue 'parole' non è evidente il motivo della traversata. L'esperienza del viaggio, in questo caso, viene sviluppata a partire da una prospettiva diversa: siamo di fronte all'appello immaginario di una madre, rivolto alla figlia che sta per lasciare il suo paese, attraverso dei mezzi di trasporto e affrontando dei rischi non meglio specificati.

\author{
Domani partirai \\ non ti posso accompagnare \\ sarai sola nel viaggio \\ io non posso venire \\ il tempo sarà lungo
}




\author{
e la tua strada incerta \\ il calore del mio amore \\ sarà la tua coperta. \\ Ho temuto questo giorno \\ è arrivato così in fretta \\ e adesso devi andare \\ la vita non aspetta \\ guardo le mie mani \\ ora che siamo sole \\ non ho altro da offrirti \\ solo le mie parole.
}

Come emerge da queste prime due strofe della canzone, e coerentemente con il genere e lo stile musicale prescelti, il linguaggio è molto chiaro ed esplicito, a basso contenuto metaforico o simbolico. Tuttavia, si nota che non viene mai nominato un toponimo che possa far dedurre quale sia il posizionamento (o per meglio dire la location) di chi parla, in questa scena immaginaria, resa efficace dalle scelte musicali di Cesare Chiodo e di Bungaro (alias Antonio Calò), e che puntano anch'esse sulla semplicità, oltre che dalle comprovate capacità performative della cantante. In altre parole, il discorso, pur nella sua chiarezza e semplicità, elude la sfera politico-sociale nonché geografica, ${ }^{24}$ e non veniamo a sapere se l' io' cantante sia da identificare con una madre eritrea, siriana, congolese, oppure, nell'eventualità, con una madre romana che saluta la figlia che emigra in Inghilterra; una calabrese che accompagna con le dovute raccomandazioni la figlia che ha trovato lavoro a Torino. Infatti, anche nel seguito, le parole chiave attorno alle quali si sviluppa il testo, rimangono riferite alla sfera sentimentale ("Rivendica il diritto ad essere felice / non dar retta alla gente / non sa quello che dice / e non aver paura / ma non ti fidare / se il gioco è troppo facile / avrai qualcosa da pagare", recita la terza strofa): una sfera semantica, questa, del resto ben sviscerata dalla musica pop italiana che annualmente si riversa sul palcoscenico del Teatro Ariston di San Remo (palcoscenico ben conosciuto anche a Fiorella Mannoia). Anche il ritornello - in Mi bemolle maggiore, come il resto della canzone - non si discosta da questa impostazione generica, sul piano semantico, da questa semplicità espressiva che lavora con pochi e ben acquisiti concetti: l'amore (della madre) qui evolve verso la sollecitazione di amare la "propria terra", e di affrontare l'incertezza del futuro con "umiltà" e con orgoglio:
Ama la tua terra
non la tradire
non badare alle offese
lasciali dire
ricorda che l'umiltà
apre tutte le porte 
e che la conoscenza

ti renderà più forte.

Ai sentimenti morali cardini sin qui elencati, si aggiungerà poi l'“onestà", nella quinta stro$\mathrm{fa},{ }^{25}$ fino all'esplosione della melodia sul Sol nell'ultima strofa, ripetuta poi una seconda volta (con variazioni testuali e con in mezzo il refrain dell'“Ama la tua terra...”): ed è qui che la scelta di una maggiore introspezione ("E io ti penserò / in silenzio"), affiancata da una breve incursione nell'ambito semantico del fantastico con "l'ora muta delle fate", viene, quasi in contrasto con il messaggio del testo, interpretato musicalmente con il fortissimo dell'effusione sentimentale, per convertirsi poi, in ultimo, in un enfatico "e griderò al mio cuore / perché tu lo possa sentire". ${ }^{26}$

Tirando le somme da quanto analizzato, potremmo dire che nonostante o forse proprio per via dell'impegno di Fiorella Mannoia sul piano sociale, nelle sue canzoni sul tema migratorio si nota a livello testuale uno sfibramento verso una serie di parole-chiave astratte, a tal punto da sfumare i contorni di una questione sociale e politica (almeno potenzialmente) ben precisa. Difatti, attraverso tali scelte testuali e musicali, la cantante riesce a non deludere, per ipotesi, né il suo pubblico di sinistra, né gli elettori medi dei partiti conservatori, di destra e populisti, che notoriamente si muovono su posizioni anti-immigrazione ma che sono ben attenti alla questione migratoria se ad essere chiamati in causa sono gli stessi italiani. Una scelta, questa, che ad una pop-star come Fiorella Mannoia è valsa l'oro, ovviamente riferito alle vendite dell'album in questione.

\section{Assalti frontali a Lampedusa}

Con l'ultimo brano qui preso in considerazione in riferimento al nostro tema, ci troviamo nuovamente in completo contrasto con quanto appena sottolineato riguardo alla tendenza, in una canzone pop come quella di Mannoia, a sfumare i contorni del reale, al fine di colpire, un po' forzatamente, la sensibilità di tutti. Si tratta di "Lampedusa lo sa", la nona traccia dell'album Profondo rosso (2011) del gruppo hip hop underground romano Assalti frontali. Attivi dal 1989, i suoi componenti, tutti sotto copertura di pseudonimi, sono emersi sull'onda del movimento studentesco della Pantera, tra l'inverno 1989 e il 1990. Il gruppo, costituitosi ufficialmente nel 1991 sotto questo nome, ha al suo attivo nove album ed è noto per il suo engagement, ma anche per non essere mai voluto entrare nei circuiti musicali più commerciali: da tanti anni si esibisce nei centri sociali romani e di altre città italiane ed è a tutt'oggi strettamente legato alla scena della contestazione sociale, giovanile e non solo (i componenti del gruppo sono ormai tutti dei quarantenni).

Dopo una intro trionfante ("Benvenuti i rifugiati, benvenuti gli immigrati, benvenuti!"), che equivale alla dichiarazione chiara della posizione politica e ideologica a partire dalla quale il gruppo intende affrontare il tema, nel seguente testo del refrain che è ritmato sullo stile del reggae, all'isola-simbolo della "grande migrazione" (Enzensberger 1993) che ha 
cambiato il volto della nostra epoca viene assegnato un ruolo diverso e nuovo: quello del testimone di questo stesso cambiamento epocale, che quindi detiene la verità e che conosce, con il beneficio della rima, qual è "la sua dignità".

\author{
La-la-Lampedusa lo sa \\ (Oh, Lampedusa lo sa) \\ La-la-Lampedusa lo sa \\ (Oh, Lampedusa lo sa) \\ Qual è la sua verità \\ Qual è la sua dignità
}

Come si potrà notare, nel testo delle strofe a seguire, da un lato si riprenderanno questi stessi concetti (il benvenuto ai boat people, l'isola-testimone, la dignità umana da essa conservata), e dall'altro si svilupperà, e questa volta con il caratteristico "declamato estemporaneo" dell'hip hop (La Via 2017, 41), un racconto del reale piuttosto articolato. Difatti, in questo brano gli Assalti frontali fanno riferimento ad un caso di cronaca risalente all'aprile 2009, quando la nave turca Pinar con a bordo 154 migranti salvati nel Mediterraneo rimase bloccata nelle acque tra Italia e Malta, e si arrivò ad un estenuante contenzioso tra i due paesi, che si rifiutarono entrambi a far sbarcare i migranti. Tale contenzioso, risolto infine soltanto con l'intervento della Corte di giustizia europea, rese evidente la totale mancanza di volontà di accogliere da parte di entrambi paesi ("quanta verità dalla nave Pinar", ripeterà più volte il cantante Militant A, nel refrain): in Italia, paese che in quel periodo vede al governo Silvio Berlusconi, con il leghista Maroni nella carica di ministro degli Esteri, scatta proprio allora il cosiddetto 'pacchetto di sicurezza' che include una legge ${ }^{27}$ che dichiara l'immigrazione cosiddetta 'clandestina' un reato, punibile con pene pecuniarie e con la reclusione. Sin dalla sua prima strofa, "Lampedusa lo sa" non solo fotografa quindi un fatto di cronaca; essa misura anche la temperatura della comunicazione politica e mediatica di quei mesi e (almeno) dei successivi due anni: comunicazione in cui (come è stato sottolineato nella parte introduttiva di questo saggio) i termini 'profugo' o 'rifugiato' vengono usati solo di rado, mentre si preferisce parlare di 'clandestini' e di 'extracomunitari'. A quest'ultimo termine viene qui associato, con chiara ironia, l'extraterrestre', in un "contro-canto" (Farinelli 2017), non solo testuale ma realizzato anche a livello musicale dalla seconda voce, che raccoglie e veicola appieno l'etica della protesta propria di questo genere di musica (oltre a riprendere una nota battuta usata già negli anni Novanta dai frequentatori di centri sociali come il Forte Prenestino di $\left.\operatorname{Roma}^{28}\right)$ :

Porta della vita, porta dell'Europa

Lampedusa ad aprile era vuota, solitaria e remota

Noi sull'isola in cento

Come un unico gruppo cantavamo nel vento

La libertà è tutto 
E non si deve sapere, non si può raccontare

La dignità è in cammino e oggi viene dal mare

C’è stato un naufragio nel viaggio

(Ma non lo diresti)

Ci sono gli extracomunitari

(E gli extraterrestri)

C'era il sole e i gabbiani, c'erano i pescecani

C'eravamo anche noi, migranti e lampedusani

Stessa felpa nera col cappuccio a salutare

Una sorella che era una pantera nera del suo cruccio

23 Aprile su quel pontile, da poco ripartito il mercantile

(Pinar)

Ora so, a Lampedusa è crudele il destino

Qui è un mare bellissimo, ma anche un mare assassino

Della funzione di testimonianza della stessa isola - che come vediamo attraverso una chiara personificazione diventa dramatis persona (e alla quale viene attribuita una dignità che gli italiani medi 'da bar' hanno da tempo persa) - si è già detto; ma nei versi appena citati emerge anche un altro aspetto dello stesso concetto di testimonianza. Infatti, è lo stesso gruppo hip hop ad essere presente sull'isola, e a testimoniare; sono gli stessi componenti del gruppo romano a mescolarsi tra i "migranti e lampedusani", per attendere il risultato della drammatica vicenda. Nella prima parte della seconda strofa, questa viene narrata nei suoi dettagli: il blocco della nave, l'intervento dei militari italiani, il tentativo di soccorso da parte di alcuni marinai, e infine le manovre volte a respingere la nave Pinar in Libia (e l'uso del verbo 'respingere', anche qui, è da considerarsi quale contro-lessico del discorso dominante di quel periodo, in cui tali pratiche venivano taciute o parafrasate - come a tutt'oggi accade - con formule eufemistiche e fuorvianti). Segue poi una seconda parte in cui si cerca di trarre le conclusioni dall'accaduto, a mo' di morale scritta a chiare lettere; una morale che è allo stesso tempo anche denuncia: perché se "non è un reato migrare", "il più assassino di tutti è senza dubbio il governo" che dichiara invece come degli 'illegali' persone che fuggono dalla fame e dalla guerra:

[...]

Lampedusa lo sa, non è un reato migrare

Stasera sarà un ponte non una barriera esemplare

Migrano balene e gli uccelli migratori

Migrano gli esseri umani e io non calmo i miei bollori

Saliti a bordo, saliti nell'inferno

Il più assassino di tutti è senza dubbio il governo 
Drammatico, infine, il racconto ritmato dell'epilogo delle vicende attorno alla nave Pinar, nell'ultima strofa. La scena è quella di un funerale, in cui il gruppo hip hop romano rimarca ancora una volta la propria partecipazione e presa di posizione, dalla parte dei "fratelli con cui condivido il grido", concludendo con una rinnovata accusa, a tutti noi, di non fare abbastanza per salvare e per accogliere, di essere quindi responsabili delle tante e troppe morti in mare (uno scenario al quale la potente outro del brano oppone infine la sua forza musicale ribelle):

$[\ldots]$

Perché questo è un mondo assurdo

Senti sulle coste l'onda d'urto

Puoi capire che mi ci catapulto

E mi suona nella testa come un antifurto

Quante volte questa porta è stata chiusa

E quante tombe senza nome a Lampedusa

Quante volte questa porta è stata chiusa

E quante tombe senza nome a Lampedusa

Benvenuti i rifugiati, benvenuti gli immigrati, benvenuti!

Benvenuti i rifugiati, benvenuti gli immigrati, benvenuti!

\section{Conclusione}

Parlare e cantare di immigrazione, anche e specie in questo nostro caso che riguarda gli sbarchi da Brindisi a Lampedusa, non può essere un semplice esercizio di espressività musicale e poetica; è sempre, anche e soprattutto, una cassa di risonanza di un posizionamento etico, di riflessioni su un'Italia e un'Europa alle prese con l'incontro/scontro con 'l'altro', è una cassa di risonanza di idee politiche e sulla società. E dunque, nel piccolo campione di testi e musiche che è stato attraversato in questa occasione è possibile osservare quanto segue: più settoriale e ristretto è il pubblico del singolo genere musicale (e del cantante o gruppo che lo frequenta), e più esplicitamente si nomina, si definisce, si usano toni indignati, dolenti, sarcastici. In un certo senso, eccetto che per il caso singolare del Canzoniere grecanico salentino, è il genere musicale a 'dettare' i contenuti e le modalità di svolgimento di questo particolare tema, a permettere o meno di prendere apertamente posizione. Infatti, nelle canzoni pop esaminate in cui vengono usati toni ora più ironici, ora più genericamente empatici verso il migrante, siamo ben lontani dagli atteggiamenti critici e accusatori, dall'ancoramento nella realtà e nella cronaca, che emergono da un genere folkloristico come quello del cantastorie così come da quello hip hop, nonostante la loro estrema diversità sul piano estetico-musicale e linguistico. Tuttavia, un elemento in comune salta agli occhi, e non è solo quello della più alta ricorrenza di brani di questo tipo negli anni più vicini a noi (e 
come evidente conseguenza dell'“effetto Lampedusa'). È che per tutti gli interpreti o autori, si tratta di incursioni sporadiche nel tema migratorio: con modalità engagé o disimpegnata che sia, da ognuno di questi artisti esso è stato finora trattato non più di una o due volte; una circostanza che difatti sorprende, perché si scontra con la triste ripetitività con cui, in questi anni, si replicano le tragedie dei naufragi, con cui si ripropongono le stesse retoriche sul piano politico-mediatico. Nonostante ciò, alla cassa di risonanza della popular music italiana nel suo insieme va il merito di illuminare sotto una prospettiva diversa una questione spesso affrontata solo attraverso slogan e cifre, o percepita come emergenza per la sola società d'arrivo; il merito, quindi, di affrontare con forza artistica, musicale e poetico-testuale una questione la cui rappresentazione è assai ardua e da sempre una sfida, ma anche (per molti) una provocazione.

\section{Note}

1 Mi riferisco in primo luogo ai docufilm La Nave dolce (Vicari 2012) e Anija la nave (Sejko 2012).

2 Arrivi e naufragi monitorati sul blog di Gabriele del Grande, Fortress Europe (http://fortresseurope.blogspot.it/), che alla pagina intitolata "La strage" ripercorre i naufragi di migranti nelle acque del Mediterraneo dal 1988, e il cui aggiornamento si è però fermato al febbraio 2016. Informazioni utili si possono inoltre ottenere dal sito www.borderline-europe.de, che include anche un progetto di approfondimento sulla situazione di profughi e migranti in Italia, oltre che dal sito ufficiale delle UNHCR (per l'Italia https://www.unhcr.it/) (consultazioni 05.02.2018).

3 Un racconto dell'avvenimento è presente in uno dei maggiori testi della letteratura italiana transculturale qual è il romanzo Madre piccola di Cristina Ali Farah (Ali Farah 2007, 15-16).

4 Con questa espressione - che indica delle scelte lessicali funzionali non tanto alla definizione chiara di qualcosa o di qualcuno, bensì strumentali alla creazione di reazioni emotive legate a delle etero-images fobiche - mi rifaccio alla terminologia imagologica usata da Daniel-Henri Pageaux in varie sue pubblicazioni (cf. soprattutto Pageaux 1994).

5 Per un'analisi sociologica compiuta sulla rappresentazione dell'immigrazione nei media italiani, si veda soprattutto Binotto/Bruno/Lai 2012, la sezione sui media in Bond/Faloppa/Bonsaver 2015, e il più recente volume collettaneo dall'approccio interdisciplinare Proglio/Odasso 2018.

6 "The theme of migrants landing on Italian shores, and more generally the dimensions of the arrival and of the length of stay of immigrants, represent one of the most characteristic elements of the entire media representation of the phenomenon. [...] landing often becomes a true media icon for the entire migration phenomenon, starting from the dynamics of strictly visual representation to the symbolic significance of the terms used by the media from a lexical-textual point of view." (Bruno 2014, 69)

7 Per il concetto di 'costruzione sociale della realtà' si veda il fondamentale testo di Berger/ Luckmann 1966.

8 A tal proposito si veda la media analysis effettuata, su un campione riguardante appunto il 2008, da Patrizia Laurano, che rivela come il termine 'clandestino' presenta una ricorrenza di gran 
lunga superiore a termini più oggettivi e rispondenti alla effettiva posizione legale dei migranti e profughi (Laurano 2012).

9 Mi riferisco principalmente ai docufilm Come un uomo sulla terra (Segre/Yimer/Biadene 2008), a Mare chiuso (Segre/Liberti 2012), oltre che al più recente lungometraggio L'ordine delle cose (Segre 2017).

10 Per le implicazioni sociologiche ed etico-politiche dell'espressione 'comunità narrativa' si rimanda a Jedlowski 2009.

11 Si tratta della decima traccia dell'album Freak (1994), su musica e testo del cantautore romagnolo.

12 Anche con questo termine, si vuole riprendere la terminologia narratologica (qui nello specifico la parola-concetto di 'storyworld'), che difatti andrà adattata alla specificità enunciatoria e al carattere ibrido della situazione comunicativa propri della canzone.

$13 \mathrm{Al}$ di là del fragile rimando nella canzone di Bersani, la televisione italiana occupa un ruolo importante nelle vicende che legano l'Albania al nostro paese sul piano dell'immaginario. Come è stato documentato da vari studi sociologici, ma anche raccontato da un film quale Lamerica di Gianni Amelio (1994) e dal romanzo I grandi occhi del mare dello scrittore di origine albanese Leonard Guaci (Guaci 2008), lungo i decenni che precedono i primi sbarchi del 1991, l'Italia fu pre-vista e sognata dagli albanesi attraverso i programmi della RAI.

$14 \mathrm{La}$ manifestazione si svolge annualmente in diverse località del Salento, concludendosi in un frequentatissimo gran finale a Melpignano (il 27 agosto).

$15 \mathrm{Va}$ inoltre ricordato che il poema Solo andata è stata una delle espressioni più fertili dell'engagement di Erri de Luca, e che ad esso si ispira anche il concept album di Gianmaria Testa intitolato Da questa parte del mare (per approfondimenti cf. Moll 2016).

16 Per la versione della canzone alla quale faccio riferimento, rinvio al sito ufficiale del gruppo: http://www.canzonieregrecanicosalentino.net/solo-andata/?lang=en (consultazione 05.02.2018).

17 Si tratta di un sintagma ricorrente nei primi capitoli del famoso romanzo di Elio Vittorini, Conversazione in Sicilia (pubblicato in pieno fascismo, per la prima volta nel 1938-1939, e nel quale emerge forte e chiara l'indignazione dell'autore verso l'ingiustizia sociale dalla quale era ed è colpita la sua Sicilia).

18 Un'analisi limpida e concisa di questo testo pasoliniano, di Peter Kammerer (Kammerer 2014), è reperibile on-line all'indirizzo http://www.centrostudipierpaolopasolinicasarsa.it/molteniblog/ la-poesia-profezia-di-ppp-tra-tragedia-e-utopia-di-peter-kammerer/ (consultazione 05.02.2018).

19 Tuttavia, la sceneggiatura del videoclip non rende giustizia al testo: la prospettiva sulle vicende è quella di un anziano pescatore italiano, che all'inizio guarda la fotografia di una donna che potrebbe essere sua madre da giovane (vestita con l'abbigliamento delle contadine del sud), e che poi vede emergere dalle acque delle persone di colore. Il loro abbigliamento anacronistico, somigliante a quello dei contadini del famoso dipinto Il quarto stato di Pelizza da Volpedo, rimanda senz'altro, sul piano semantico, al tono arcaicizzante del testo di De Luca. I naufragi giungono a riva e scompaiono, ma in ultimo il pescatore si lancia nelle acque per salvare una donna rimasta indietro: una donna che, una volta salva e giunta a riva, mostra lo stesso volto della donna sulla fotografia che il pescatore aveva tenuto in mano. Nonostante il corretto collegamento con il pas- 
sato povero degli italiani popolo di migranti, in questa trasposizione visiva dei versi di De Luca si coglie l'incapacità di rovesciare la prospettiva, e di assumere lo sguardo di quel 'noi' che lo scrittore, invece, ha raccolto con forza nel suo componimento, e che trova un ulteriore potenziamento nella cassa di risonanza di questa interpretazione musicale.

20 Per i riferimenti da parte di Geraci ai suoi stessi 'auctores', si rimanda alla sua canzone "Io cantastorie".

21 "La Sicilia per noi cantastorie non è l'isola. È la piazza del mondo", scrive Geraci sul suo blog (sul quale sono disponibili numerosi testi di canzoni e alcuni file audio): http://www.geracicantastorie.com/ (consultazione 05.02.2018); la versione audio della canzone qui analizzata è invece disponibile solo su YouTube: https://www.youtube.com/watch?v=cdms0fJEuOw (consultazione 05.02.2018).

22 Si va strincennu puru lu parrari supra lu ponti di dda navi persa, sutta non hannu ciatu pi ciatari 'ffuga la nafta ogni parola persa.

$\mathrm{Cu}$ è svigghiu ancora pensa a lu caminu

chi fici pi fujri di la guerra,

d'a fami dulurusa e d'un cainu

chi lu tineva cu lu mussu 'n terra.

E fora 'u mari 'nzonna la curuna,

li stiddi di l'Europa assai stramani,

chi surgi e a tutti l'amuri ci duna,

na tavula cunzata e un pezzu 'i pani.

...e inveci è scuru,

non puoi scappari,

lu mari è muru

senza lampari,

sulu 'u muturi

nta l'ariu forti

svapura uduri

comu la morti.

23 Ad esempio, Mannoia è stata madrina del Festival Sabir che si è svolto nell'ottobre 2014 sull' isola di Lampedusa, e nel quale sono stati coinvolti vari artisti e intellettuali, italiani e immigrati.

24 Tale dimensione viene elusa anche in "Se solo mi guardassi", canzone nella quale l' io' cantante è da identificare con un diverso, un migrante si potrebbe supporre, che chiede all'autoctono una visibilità normalmente negata.

25 Lo sai che l'onestà

non è un concetto vecchio

non vergognarti mai

quando ti guardi nello specchio 
non invocare aiuto nelle notte di tempesta

e non ti sottomettere tieni alta la testa.

$26 \mathrm{Ed}$ io ti penserò in silenzio

nelle notti d'estate,

nell'ora del tramonto

quella muta delle fate

e parlerò al mio cuore

perché, domani partirai

in silenzio

ma in una notte di estate

io ti verrò a cercare

io ti verrò a parlare

e griderò al mio cuore

perché... tu lo possa sentire

si, lo possa sentire...

tu lo possa sentire.

27 Si tratta della legge n. 94 del 2 luglio 2009.

28 Ancora sui legami tra hip italiano e culture della protesta si veda (per il caso degli Assalti frontali e non solo) Ivic 2010, che raccoglie anche una serie di interviste agli esponenti principali del genere, attivi nelle varie regioni italiane.

\section{Bibliografia}

Ali Farah, Cristina: Madre piccola. Roma: Frassinelli, 2007.

Berger, Peter L. / Luckmann, Thomas: The Social Construction of Reality. A Treatise in the Sociology of Knowledge. New York: Doubleday, 1966.

Binotto, Marco / Bruno, Marco / Lai, Valeria (ed.): Gigantografie in bianco e nero: ricerca su sicurezza, immigrazione e asilo nei media italiani. Raleigh NC: Lulu Press, 2012.

Bruno, Marco: “The Journalistic Construction of 'Emergenza Lampedusa': The 'Arab Spring' and the 'Landings' Issue in Media Representation of Migration”. In: Bond, Emma / Bonsaver, Guido / Faloppa, Federico (ed.): Destination Italy. Representing Migration in Contemporary Media and Narrative. Oxford / Bern: Peter Lang, 2015, 59-84.

De Luca, Erri: Solo andata. Righe che vanno troppo spesso a capo. Milano: Feltrinelli, 2005.

Enzensberger, Hans Magnus: La grande migrazione. Torino: Einaudi, 1993.

Fanelli, Antonio: Contro canto. Le culture della protesta dal canto sociale al rap. Roma: Donzelli, 2017.

Fanon, Frantz: I dannati della terra. Ed. L. Ellena. Trad. C. Cignetti. Torino: Einaudi, 2007.

Giovannetti, Paolo: Dalla poesia in prosa al rap. Tradizioni e canoni metrici nella poesia italiana contemporanea. Novara: Interlinea, 2008.

Guaci, Leonard: I grandi occhi del mare. Nardò: Besa, 2008.

Ivic, Damir: Storia ragionata dell'hip hop italiano. Roma: Arcana, 2010. 
Jedlowski, Paolo: Il racconto come dimora. 'Heimat'e le memorie d'Europa. Torino: Bollati Boringhieri, 2009.

Kammerer, Peter: "La profezia di Pier Paolo Pasolini tra tragedia e utopia". In: Centro studi Casarsa Pier Paolo Pasolini (dicembre 2014), http://www.centrostudipierpaolopasolinicasarsa.it/molteniblog/la-poesia-profezia-di-ppp-tra-tragedia-e-utopia-di-peter-kammerer/ (consultazione 30.01.2018).

Laurano, Patrizia: "Arrivi, sbarchi, rimpatrio". In: Binotto, Marco / Bruno, Marco / Lai, Valeria (ed.): Gigantografie in bianco e nero. Raleigh NC: Lulu Press, 2012, 121-128.

La Via, Stefano: Poesia per musica e musica per poesia. Dai trovatori a Paolo Conte. Roma: Carocci, 2017.

Moll, Nora: "Das Migrationsthema bei Gianmaria Testa: zur ethischen und ästhetischen Hinterlassenschaft des Liedermachers aus dem Piemont". In: ATeM 1 (2016), https://atem-journal. com/ojs2/index.php/ATeM/article/view/1657 (consultazione 30.01.2018).

Pageaux, Daniel-Henri: "Images". In: Pageaux, Daniel-Henri: La littérature générale et comparée. Paris: Armand Colin, 1994, 59-76.

Proglio, Gabriele / Odasso, Laura: Border Lampedusa. Subjectivity, Visibility, and Memory in Stories of Sea and Land. London: Palgrave Macmillan, 2018.

\section{Discografia}

Assalti frontali: Profondo rosso. Assalti frontali B008RY5WSG, 2011 (CD).

Bersani, Samuele: Freak. Pressing B00005S21K, 1994 (CD).

Canzoniere Grecanico Salentino: "Solo andata" (1994). In: http://www.canzonieregrecanicosalentino. net/solo-andata/?lang=en (consultazione 30.01.2018).

Geraci, Mauro: "Lampedusa" (2014). In: http://www.geracicantastorie.com/ (consultazione 30.01.2018). Mannoia, Fiorella: Sud. Sony Music/Oyà B007D5JWWM, 2012 (CD).

\section{Filmografia}

Amelio, Gianni (reg.): Lamerica. Italia, 1994.

Segre, Andrea / Yimer, Dagmawi / Biadene, Riccardo (reg.): Come un uomo sulla terra. Italia, 2008.

Segre, Andrea / Liberti, Stefano (reg.): Mare chiuso. Italia, 2012.

Segre, Andrea (reg.): L'ordine delle cose. Italia, 2017.

Sejko, Roland (reg.): Anija la nave. Italia, 2012.

Vicari, Daniele (reg.): La Nave dolce. Italia, 2012. 


\section{Sitografia}

Borderline Europe: http://www.borderlineeurope.com/ (consultazione 30.01.2018).

Fortress Europe: http://www.fortresseurope.blogspot.it/ (consultazione 30.01.2018).

UNHCR Italia: https://www.unhcr.it/ (consultazione 30.01.2018). 